\title{
MATHEMATICAL MODELLING PROCESS OF TUNGSTEN REDUCTION BY HYDROGEN \\ OF THE FLUORIDES
}

\author{
Roman Brendakov ${ }^{1, *}$, Alexander Shvab ${ }^{1}$, and Vladimir Brendakov ${ }^{2}$ \\ ${ }^{1}$ National Research Tomsk State University, 634030 Tomsk, Russia \\ ${ }^{2}$ Seversk Technological Institute NRNU MEPhl, 636070 Seversk, Russia
}

\begin{abstract}
The process of tungsten fluorides reduction by hydrogen is a component part of Fluoride technology of tungsten conversion. Nowadays the researchers are definitely interested in studying this process. It is connected with common use of metal tungsten products in different sectors of the economy, which is the result of unique qualities of this metal. With the help of physical and mathematical modelling of the process of tungsten hexafluoride reduction by hydrogen, it becomes possible to create an import substitution technology of metal tungsten conversion. Fluoride technology of tungsten conversion allows putting different coverings and make tungsten products of different shapes, which is impossible to get traditionally. The process of tungsten fluorides reduction by hydrogen can be referred to CVD processes (Chemical Vapor Deposition). Common use of CVD technologies for getting metal products and coverings is limited by definite problems, connected with access difficulties to initial components of research and the lack of information about their basic thermal characteristics. Therefore, mathematical description of the initial components mass-moving process, which provides with optimal value of their concentration in gas flow and in precipitation zone, is a question of current importance.
\end{abstract}

The process of tungsten fluorides reduction by hydrogen is realized according to chemical reaction, represented by a formula

$$
W F_{6}+3 H_{2}=W+6 H F
$$

Mathematical model of reduction process should describe non-stationary processes of heat and mass exchange when two-component mixture moves in the reactionary zone. The flat channel in the article is described and presented in the figure 1, in which the mixture of two gaseous substances, tungsten hexafluoride and hydrogen enters vertically through the bottom entry section at the $T_{0}$ temperature. The balance of these gases concentration is consistent with stoichiometric coefficient in equation (1). This mixture is considered to be the first component with mass concentration equal 1 at the entry section. The second component in this working zone is fluoric hydrogen, which shows up as a result of reaction

*Corresponding author: brend989@gmail.com 
(1) near the heated bottom layer, situated on the left-side boundary line with the constant $T_{W}$ temperature. It is possible to neglect the metal thickness, which shows up on the bottom layer, as it has a small size.

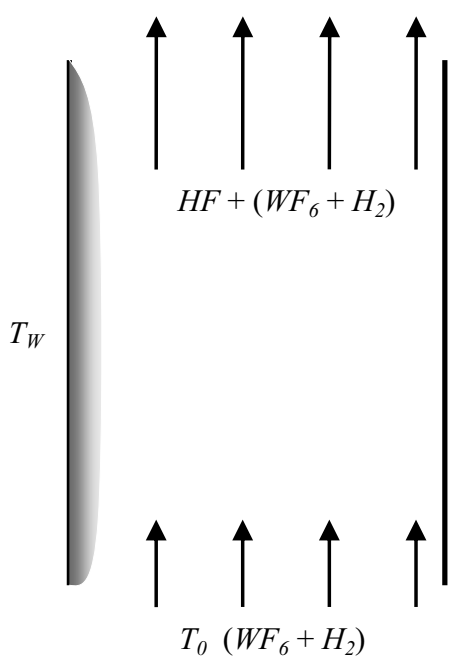

Fig. 1. Working zone scheme.

Taking into consideration all the allowances, the equations of convective heat- and mass-moving in the flat channel are:

$$
\begin{gathered}
c \rho(\partial \theta / \partial \tau+u \partial \theta / \partial z)=\partial(\lambda \partial \theta / \partial z) / \partial z+\partial(\lambda \partial \theta / \partial x) / \partial x \\
\partial C / \partial \tau+u \partial C / \partial z=\partial(D \partial C / \partial z) / \partial z+\partial(D \partial C / \partial x) / \partial x \\
\theta=\left(T-T_{0}\right) /\left(T_{w}-T_{0}\right)
\end{gathered}
$$

where $c, \rho, \lambda$ are thermal capacity, density and thermal conductivity of the gas flow accordingly; $C, D$ is mass concentration and diffusion coefficient of key flow component $\left(\mathrm{WF}_{6}+3 \mathrm{H}_{2}\right)$.

For quasi-stationary speed type of stabilized gas flow in the flat channel, the expression is the following, taking into account formula (1):

$$
\begin{gathered}
u=6 \bar{u}\left[x / x_{\max }-\left(x / x_{\max }\right)^{2}\right] ; \\
\bar{u}=(3-C) \bar{u}_{0} / 2 ;
\end{gathered}
$$

$\bar{u}$ is a gas flow speed, averaged through a cross-section, $x_{\max }$ is a value of a crossed coordinate on the right border of the area, $\bar{u}_{0}$ is an averaged speed through a cross-section.

Therefore, the mathematical model of the process of tungsten fluorides reduction by hydrogen is a system of varied equations in partial derivatives (2), transport equation type, written for expansible temperature $\theta$ and concentration of the key component of mixture $C$ can be solved computationally under certain limited circumstances known. Taking into consideration the equation of chemical reaction (1), it is enough to know the algorithm of calculation the speed of the reaction of reduction $v_{w}$ to know the values of mixture components concentration on the surface of the bottom layer through the characteristics of the flow in the calculated area, completing the task this way.

In the work (1) the formula is offered to find the speed of tungsten concretion from the mixture $\mathrm{WF}_{6}+\mathrm{H}_{2}+\mathrm{HF}$ at the atmosphere pressure of 


$$
v_{w}=\frac{3,6 \cdot 10^{19} N_{H_{2}} N_{W F_{6}} e^{-\frac{58500}{R T}}}{\left(\frac{1}{\rho}+\frac{21500}{\sqrt{T}} N_{H_{2}} e^{\frac{840}{R T}}+\frac{25600}{\sqrt{T}} N_{W F_{6}} e^{\frac{6700}{R T}}+\frac{20000}{\sqrt{T}} N_{H F} e^{\frac{4180}{R T}}\right)^{2} T^{3}}
$$

While deducing this formula there have been made some allowances.

First of all, it is considered, that the process of tungsten concretion is done at the constant temperature. Second, the adsorptive balances in the system are reached faster, than the reaction goes, which means, that at any moment there is a certain balance between partial pressure of the component of the reaction in the gas phase and the part of reactionary surface, occupied by its molecules. The comparison of big number of experimental data $[2$, 3 , 4] with theoretical calculation concerning this equation revealed a satisfactory correspondence in a wide interval of process parameters varying, though it shouldn't be omitted, that it is only right in the kinetic part of the process. In the diffusive part, as expected, experimental data is rather lower, than that in the formula above.

Taking all the limitations and allowances into account, there has been made a test calculation of the changing of tungsten layer thickness through the length of bottom layer in comparison with the experiment $\bullet$ [1]. The result is given in the figure 2 .

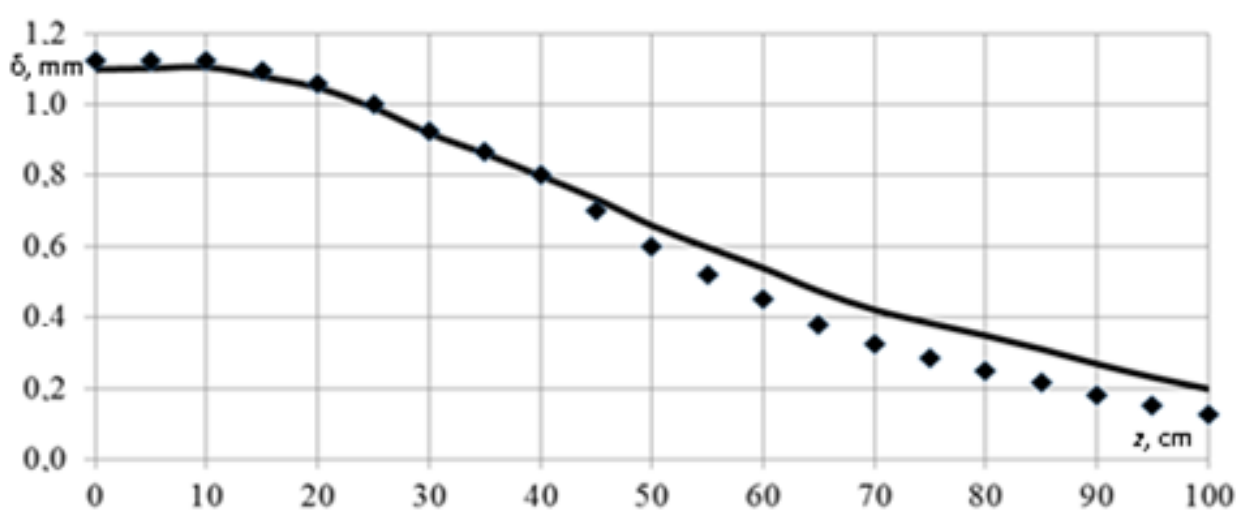

Fig. 2. The changing of tungsten layer through the bottom layer length.

A good correspondence of calculation and experimental data allows making a conclusion about the adequacy of created model. This model allows making numeral research of the optimization of the process of refractory metal fluorides reduction.

\section{References}

1. Yu. M. Korolev, V. I. Stolyarov, Restoration of fluorides of refractory metals hydrogen (Metallurgy, Moscow, 1981)

2. U. N. Golovanov., S. N. Kadimet., A. I. Krasovskii etc. The electronic technics. Technology, production process and equipment 43 (1971)

3. V. A. Neiberlein, J. Amer. Ceramic. Soc. Bulletin 48 (1965)

4. I. B. Averin, U. P. Plotnikov, Mech. Eng. 107 (1979) 